\title{
Téoros
}

Revue de recherche en tourisme

\section{Formation touristique : le choix des moyens}

\section{Michel Tiard}

Volume 8, numéro 1, mars 1989

France-Québec

URI : https://id.erudit.org/iderudit/1080360ar

DOI : https://doi.org/10.7202/1080360ar

Aller au sommaire du numéro

\section{Éditeur(s)}

Université du Québec à Montréal

\section{ISSN}

0712-8657 (imprimé)

1923-2705 (numérique)

Découvrir la revue

Citer cet article

Tiard, M. (1989). Formation touristique : le choix des moyens. Téoros, 8(1),

37-40. https://doi.org/10.7202/1080360ar d'utilisation que vous pouvez consulter en ligne.

https://apropos.erudit.org/fr/usagers/politique-dutilisation/ 
La formation est un secteur d'activités qui passionne et la majorité des lecteurs d'ESpacES est directement concernée par la formation touristique. Nombreux sont ceux par exemple qui ont franchi, à un moment donné, la barrière séparant les "formés" des formateurs, en formation initiale ou continue.

Les formateurs, qui exercent un métier de ce thème sont fréquents. Ils sont rédigés sur un registre qui va de l'auto-satisfaction au désenchantement, et il faut reconnaître que ce dernier sentiment ainsi qu'une perception globalement négative de la formation touristique ont largement cours actuellement.

Plusieurs facteurs peuvent expliquer cette situation, en dehors des défauts et qualités intrinsèques des formations en question. Certains ne sont pas spécifiques au secteur touristique:

- de façon générale, le fort taux de chômage en France, et surtout parmi les jeunes, ne peut conduire, tant qu'il durera, qu'à deconsiderer bien des formations initiales. Il est difficile de faire la part des choses dans ce cas: le chômage est-il dû principalement ầ une formation inadaptée ou à un manque de création d'emplois? Une formation pointue n'aurait-elle pas pu être elle-même créatrice d'emplois, donner des idées aux employeurs potentiels? La dévalorisation des fonctions d'enseignant et de formateurs tient aussi à l'existence d'une masse importante de chômeurs;

- le "traitement social" du choimage, les dispositifs de type TUC ou SIVP, ont aussi contribué à l'image négative des formations. Pour beaucoup, les stages ne sont qu'une "salle d'attente" de la vie active, un moyen de déguiser les statistiques du chômage: de là̀ à penser qu'ils ne sont que cela... L'INSEE (dans son no 216 d'Économie et Statistiques de décembre 1988) donne des informations précieuses sur ce point. la communication, Ecrivent et les articles sur

Certains facteurs sont spécifiques au secteur touristique:

- la formation touristique, c'est d'abord une activité de service destinée à améliorer une autre offre de service, avec toutes les difficultés qui en résultent pour évaluer les résultats des formations ne préparant pas également à des examens nationaux. Bien entendu, les taux de réussite à un examen ne sont qu'un indicateur parmi d'autres, permettant de juger le centre de formation mais non l'adéquation globale de la formation aux besoins du secteur d'activité. Nous nous heurtons ici encore au manque de données statistiques sur certaines activités de services et aux problèmes de fiabilité de celles qui existent;

- il n'existe pas en France UN centre de formation touristique qui s'est imposé et sert de référence, une grande école nationale de tourisme, mais des établissements d'enseignement et des centres de formation, publics et privés, atomisés et finalement de faible envergure. Les initiatives sont éparpillées, les moyens mis en oeuvre rendent les actions plus ou moins crédibles d'où un sentiment d'insatisfaction diffus mais réel chez les formateurs, les stagiaires, scolaires et étudiants et a fortiori chez les employeurs potentiels;

- I'intérêt des entreprises touristiques pour la formation sous toutes ses formes est inégal alors que la volonté des établissements de formation d'établir des liens avec ces entreprises semble s'être généralisée aujourd'hui. De ce point de vue on ne peut pas dire qu'une reconnaissance mutuelle soit effective: nous retrouvons une coupure dans les appellations "professionnels "7/ "professeurs" ou "formateurs" désignant deux catégories bien compartimentées, la deuxième n'étant pas encore totalement acceptée comme "professionnels de la formation ". L "idée suivant laquelle un formateur est un professionnel qui $n$ 'a pas vraiment réussi survit encore pour diverses raisons:

- de façon générale, nous pouvons dire que les débats, polémiques et, dans le meilleur des cas, les échanges d'idées sur la formation, rejoignent tout ce qui a pu être dit jusqu'à présent sur les lacunes constatées en France en matière:

- d'observation ceonomique et statistique du tourisme,

* Michel Tard est professeur au Lycée d’Hátellerie et de Tourisme de Saint-Quentin-en-Yvalynes at au C.E.S.T.

- de centres documentaires,
- d'information touristique à caractère technique et professionnel.

De façon évidente il existe des interactions entre ces outils des entreprises touristiques et la formation, pouvant conduire à des synergies ou des interactions négatives.

Dans la mesure où une réflexion sur les moyens réels disponibles pour assurer la formation initiale et continue dans le tourisme est indispensable nous aborderons les points suivants:

- les centres de formation,

- les programmes de formation,

- les formateurs,

- les stages dans les entreprises.

\section{Les centres de formation touristique}

La singularisation des formations touristiques en France est relativement récente, tant en ce qui concerne l'élaboration de programmes spécifiques que la création de centres de formation. Quelques dates et faits significatifs:

1960 - première version du Brevet de Technicien Supérieur de Tourisme, issu d'un brevet professionnel attribué à partir de 1946 ,

1961 - création du Centre d'Êtudes Supéricures de Tourisme par Louis Burnet,

1969 - apparition du Brevet de Technicien du Tourisme.

Un cocktail fait d'initiatives d'individus avertis et de la volonte des pouvoirs publics (1'Etat aménageur des annés 60) a donné alors à l'Éducation Nationale un rôle de pionnier en ce domaine.

Aujourd'hui, ces formations coexistent le plus souvent avec d'autres filières dans un même tablissement:

- hôtellerie, formations diverses du tertiaire, langues étrangères appliquées (L.E.A.), droit, etc... pour le secteur public;

- BTS du tertiaire, formations commerciales supérieures, etc... pour le secteur privé.

Si l'on se réfere à la taille de ces établissements, la ventilation de ceux-ci peut s'établir ainsi: 
- des établissements de petite taille se consacrant spécifiquement à des formations touristiques, avec des couts de structure relativement élevés par rapport au "debit" d'elèves, étudiants, stagiaires;

- des établissements plus importants intégrant les formations touristiques comme une diversification de leurs activités; il semble que ce soit une tendance à l'heure actuelle, justifiée pour les filières touristiques, par une mise à disposition de moyens plus importants;

- le créneau "Établissement important avec formation touristique exclusive " n'est pas encore occupé pour le moment. Il devrait l'être en 1990 avec l'ouverture à Marnela-Vallee de l" "European Instime of Tourism Management" pouvant accueillit "350 à 400 étudiants dans le cadre de promovions d'environ 200 personnes en formation permanente ef environ autan en formation continue". (Interview de Joseph Olivereau par I'Écho Touristique)

Cette " grande ecole prive internationale de tourisme" est qualifiée d" "Ecole de finition" par Joseph Olivereau qui prévoit un recrutement international à un niveau grande école avec des frais de scolarité d'environ $140.000 \mathrm{~F} / \mathrm{an}$

La disproportion entre ce projet, apparemment bien avancé, et la situation présente fait ressortir les faiblesses des ressources traduites par ces deux cas de figure:

- tous les moyens, financiers et humains, dans des établissements et centres de formation... disposant de moyens limités:

- des moyens, dont les criteres de répartition ne favorisent pas toujours les filières touristiques, dans les autres établissements à formations diversifices.

Les explications envisageables sont nombreuses pour permettre de comprendre cet emiettement des moyens. Nous pourrions ainsi évoquer un éparpillement de la taxe d'apprentissage, un marché non solvable de la formation continue généré par l'ensemble des entreprises non assujetties à la participation à la formation professionnelle. Et puis, notons que les syndicats d'employeurs ou de salariés sont à l'image des entreprises touristiques: atomisés - et, lorsqu'ils existent, dans l'impossibilité de se déclarer et surtout de se faire connaitre comme représentatifs d'un vaste secteur. La notion de lobby, de groupe de pression crédible et pouvant influer sur le développement de la formation touristique, est plus un thème de réflexion qu'une réalité.

La dispersion des établissements techniques et universitaires, centres de formation initiale et continue, publics et prives, pourrait représenter un atout par le foisonnement des initiatives, par la complémentarité des spécialisations, la multiplication des "passerelles" entre formations. Mais on ne peut pas dire que cette dispersion soit le résultat d'une concertation. Au niveau universitaire, les cen- tres de formation benéficient souvent d'une ETIQUETTE, c'est-à-dire qu'une dominante lui est attribuée: "gestion", "droit", "langues". "marketing", "geographie", etc... Mais est-ce une spécialisation réelle basée sur le poids de certains enseignements? Et dans ce cas, pouvons-nous envisager une complémentarité récelle des formations ou une complémentarité d' image des centres de formation? Dans ce cas, la multiplication des filières universitaires aboutirait plutồt à la duplication de formations similaires, avec des recrutements d'étudiants à une échelle régionale, qu'à la crétation de formations originales impliquant un recrutement national ou même international.

Ce dernier point, cette "preuve par l'étranger", constitue un theme d'analyse digne d'intérêt:

- des centres de formation performants, $c^{7}$ est une adaptation aux besoins nationaux en matière de qualification mais c'est aussi la réception d'ćtudiants et stagiaires étrangers done une exportation de savoir-faire, avec des retombées économiques supplémentaires à terme;

- des centres de formation inadaptés, ce sont des activités touristiques en France pouvant crét des emplois... aux PaysBas, en RFA, par exportation d'une maind'oeuvre mieux formée, ce sont également des étudiants et stagiaires étrangers drainés par d'autres pays que la France.

L'ampleur des enjeux, parfois extrahexagonaux, ne doit pas faire oublier certaines contraintes, quelquefois mal vécues, liées à la politique (ou pire, aux modalités) de recrutement des centres de formation. Les problèmes liés au recrutement semblent plus toucher le privé que le public, le technique que le supérieur, la formation initiale que la formation continue. Si l'on prend l'exemple de l'hôtellerie et de la restauration, on peut penser que les candidats aux formations se sont au départ pré-sélectionnés eux-mêmes: la part de rêve attachée à des métiers dont l'image est plutôt "dure" semble réduite.

Par contre, les métiers du tourisme et des loisirs souffrent de la persistance de clichés sur lesquels nous ne nous appesantirons pas, sauf pour noter qu'ils peuvent aussi marquer les personnels charges de l'orientation scolaire.

Ne pas sélectionner sur des critères de niveau et de motivation sérieuse conduit à des errements, à tous les niveaux de formation considérés:

- remise à niveau obligatoire des scolaires ou stagiaires;

- en conséquence accroissement de la part "culture generale/connaissances de base " par rapport à la formation spécifiquement touristique;

- à terme, afflux toujours plus grand de "marginalises de l'enseignement"

et le cycle reprend.
C'est pour cela aussi que l'on peut juger de façon positive, comme un garde-fou et une fenêtre sur le monde, la cohabitation des filières de formation touristique et des filières de type "management" ou autres; C'est une façon de stimuler et d'ouvrir l'esprit des "formes"s... et des formateurs.

\section{Les programmes de formation}

Ceci n'est pas propre au secteur qui nous intéresse mais les programmes, plus spécialement en formation initiale, ne sont pas vraiment connus des employeurs, ni même des ćlèves et étudiants avant le début de leurs études. Il est difficile d'avoir une vision globale de ces programmes de formation touristique en France et généralement la connaissance que nous avons des formations étrangères est fragmentaire. Aurait-on plus confiance dans les centres de formation eux-mêmes que dans les programmes élaborés et dans leur application?

L'elaboration et l'adaptation des programmes pâtissent des lacunes de l'information sur l'évolution des activités touristiques et de loisirs. Quand la volonté d'adaptation existe, elle est freinée: faute de données globales suffisantes, qualitatives et quantitatives, certaines conclusions reposent sur des impressions ou la théorisation d'expériences personnelles. Ce manque de donnees implique donc l'engagement de procedures assez lourdes pour la mise au point de programmes de formation initiale dans l'enseignement public. Par contre, en matière de formation continue, ainsi que dans le secteur privé en général, les programmes peuvent être issus d'études préalables plus légères ou de la simple identification d'opportunites commerciales.

L'adaptation des formations pose des problèmes d'échéance et donc de "rodage" des programmes: faut-il envisager le court ou le long terme? Comment concilier le double imperatif des besoins à court terme de l'employeur "lambda" et les besoins à moyen et long termes du pays, ceux que prend en compte l'Éducation Nationale? Nous pourrions résumer ainsi ces deux catégories d'objectifs:

- une formation d'adaptation, préparant aux diverses évolutions prévisibles ou non du tourisme et des loisirs,

- une formation de "finition", prise en charge par les centres de formation et les entreprises, séparénent ou conjointement.

Ne pas distinguer ces deux objectifs conduit parfois des employeurs à négliger l'intérèt de certaines formations. Mais il faut aussi que les programmes intègrent la possibilité de formations complémentaires de finition. plus spécialisées. Des initiatives ont été prises depuis deux ou trois ans: 3ème année de spécialisation post-BTS dans des écoles privées, formation en "Communication internationale" au Lycée d'Hôtellerie et de Tourisme de Saint-Quentin-en-Yvelines, 
"Licence de gestion des entreprises touristiques "' aे Nanterre/Paris $\mathrm{X}$, etc...

De mếme qu'il y a peu de centres de formation specifiquement "tourisme", quelle est la part réelle des blocs horaires "tourisme" dans les programmes? Si l'originalité du tourisme... c'est le TOURISME, une demande spécifique, des entreprises fonctionnant avec des contraintes particulières, etc... combien de formations en tiennent compte? Ici diverses contraintes interviennent, nous les avons déjà évoquées précédernment en matière de sélection et de recrutement, nous pouvons y ajouter celle de l'utilisation des formateurs disponibles.

La modernisation des formations touristiques posera peut-être des problèmes d'identité de ces formations: comment tirer le contenu de ces programmes vers des techniques et des matières "performantes" sans que ceux-ci perdent leur âme, c'est-à-dire en d'autres termes sans qu'ils constituent au bout du compte des doublons de formations plus spécialisées (marketing, gestion, informatique, etc.) perdant du même coup leur avantage concurrentiel: gestion, marketing, informatique + tourisme. Cet élément est essentiel pour une raison de crédibilité: la perte d'une spécificité "tourisme" dont la valeur serait reconnue, ne ferait qu'accentuer le poids des filières de formation extérieures au tourisme. Pour éviter cet écueil, plusieurs précautions paraissent nécessaires:

- Diminuer la part des cours et actions de simple INFORMATION au profit d'une véritable action de formation visant à l'acquisition de concepts et de méthodes, de capacité d'analyse et de modification du comportement. Cette appropriation individuelle d'un savoir-faire légitime plus qu'autre chose les formations initiale et continue. Il est vrai qu'en période d'évolution intense et rapide des activités de tourisme et de loisirs en France et en Europe, la synthèse d'informations et leur transmission aux élèves et stagiaires semble indispensable. Mais ne s'agit-il pas d'une solution de facilité à court terme permettant juste de suppléer à l'effort individuel d'information des étudiants et stagiaires?

- Veiller à une approche GLOBALE et non SECTORIELLE de la formation, ce qui est toujours plus nécessaire à mesure que l'on prépare à un niveau de qualification plus élevé. En effet, si les pratiques professionnelles du tourisme ne sont considérées que comme des pratiques et des techniques banalisées, une formation "tourisme" spécifique peut-elle se jus= tifier?

- Intégrer dans les programmes de formation initiale des possibilités d'innovation, d'adaptation, en respectant l'esprit et non la lettre de ces programmes. Ceci est lié à une évaluation constante laquelle marque pour le moment la frontiere entre la formation initiale et continue.
- Prévoir des passerelles entre les matières enseignées afin de faire percevoir la cohérence des programmes. Là où les formateurs perçoivent justement cette cohérence, les "formes' " ne ressentent peutêtre dans certains cas que la lourdeur et l'apparente répétitivité de l'ensemble?

Tout ceci rend encore plus souhaitable un investissement réel des professionnels du tourisme et des loisirs dans la mise au point des programmes, sans confondre confrontation des points de vue et affrontement. Au-delà de la collaboration sous forme de conférences, acceptation de stagiaires exprimant une caution mutuelle des formateurs et praticiens, des possibilités d'élaboration conjointes des programmes de formation existent, certaines ont été exploitées, d'autre pas.

\section{Les formateurs}

Un des soucis de l'Éducation Nationale ayant conduit à la mise en chantier d'un nouveau Brevet de Technicien Supérieur de Tourisme pourrait, semble-t-il, être illustré par cette question: " Est-il viable, aujourd'hui, d'être professeur de TOURISME?" (ct à plus forte raison dans 5 ou 10 ans...). Cette approche pourrait être étendue à tous les niveaux de formation touristique.

Les généralistes de la formation touristique ont marqué une époque, il y a 20 à 25 ans, quand cette formation cherchait son autonomie par rapport aux autres filières. Mais, depuis, la nécessité d'une plus grande spécialisation est apparue, ne serait-ce que pour permettre à chaque formateur d'assimiler les évolutions complexes des activités touristiques et de loisirs dans un environnement toujours plus international. Pour de nombreux formateurs, des exigences nouvelles se manifestent:

- un fort ancrage disciplinaire tout en maîtrisant une vision globale du tourisme et des loisirs;

- une spécialisation venant se greffer sur une bonne formation de base, laquelle garantit une qualité certaine de la transmission des connaissances;

une bonne maîtrise des niveaux MICRO et MACRO, de l'entreprise et de l'ensemble de son environnement:

une ouverture professionnelle se traduisant par des contacts permanents avec toutes sortes d'organismes touristiques.

Le profil des formateurs n'est pas le seul élément à retenir dans les transformations de ces dernières années. Les besoins quantitatifs ont aussi profondément changé le paysage de la formation touristique.

Au fil des annees, les formations longues ont eu un effet incitatif sur les niveaux immédiatement supérieurs:

- mise en concurrence des détenteurs du BTS de Tourisme (ainsi que ceux qui ont échoué à cet examen) avec les détenteurs du seul Brevet de Technicien;
- alimentation d'une partie des filières "Licence de Tourisme" par des BTS;

- développement des formations à bac + 4 ou 5.

Voici pour la multiplication des formations par strates, laquelle s'est effectućc en conjonction avec d'autres processus: croissance du secteur privé, diversification de la formation continue et régionalisation. Il en résulte une abondance des formations dont le taux d'accroissement a été plus rapide que celui des formateurs en mesure de les prendre en charge.

Exigences nouvelles en matière de compétence et centres de formation en surnombre par rapport à la population de formateurs constituent un véritable goulet d'étranglement.

Autre distorsion relevế, celle qui existe entre le niveau de compétence théoriquement nécessaire et les perspectives de carrière professionnelle des formateurs. On peut même dans certains cas d'impasse professionnelle. les évolutions de carrière profitables impliquant une sortie des filières touristiques assez fréquemment.

Nous terminerons cette partie par la formation continue des formateurs eux-mêmes; elle représente un besoin ressenti par beaucoup d'entre eux: nécessité d'une mise à jour régulière de la connaissance des activités touristiques et de loisirs, periodes de recyclage sous forme de stages longs, etc... La seule formation initiale des enseignants et formateurs en général ne suffit plus. Diverses solutions semblent correspondre à ce besoin exprimé, par exemple:

- des stages courts annuels en entreprise. de préférence dans des entreprises performantes, dans les secteurs les plus divers et dans des régions touristiques en pointe du point de vue de l'offre et de la commercialisation:

- par période des stages longs plutôt qu'une multiplication de stages courts, dans le cadre de la spécialisation du formateur.

\section{Les stages en entreprise}

Ingrédients importants des formations initiales et de longue durće, les stages en entreprise sont l'expression

- d'une collaboration entre l'entreprise et les centres de formation, l'entreprise étant elle-meme lieu de formation;

- d'un premier test des formations "en grandeur nature";

- d'une mise à l'épreuve des motivations des scolaires et stagiaires.

Mais il y a certainement des BONS et des MAUVAIS stages. En premier lieu, les stages ont une incidence globale sur divers secteurs touristiques: si l'on s'en tient au seul BTS, de 4.000 à 5000 élèves effectuant un stage de 2 à 3 mois, cela représente de 8.000 
à 15.000 mois de stage par an, privilégiant les agences de voyages, les villages de vacances. De ce fait, il y a interaction entre les stages et l'emploi saisonnier, de nombreuses possibilités de "dérapage" entre la notion de stagiaite et de saisonnier. Et puis il y a vraisemblablement les " $B O N S$ " et les "MONS BONS" stagiaires: Pour reprendre l'exemple du BTS de Tourisme, il ne faut pas négliger le fait qu'en fin de première année de cours, donc au milieu d'un cycle non achevé, de 60 à $70 \%$ des stages sont effectués par des stagiaires qui échoueront à l'examen; ceci pose un problème d'image pour la formation en elle-même auprès des entreprises et maitres de stages.

Les particularités des entreprises touristiques et de la forte saisonnalité de l'activité en France peuvent être à l'origine de quelques inconvénients:

- présence simultance de trop nombreux stagiaires dans une petite entreprise;

- niveau d'activité trop, ou trop peu, élevé pendant les vacances scolaires où se déroulent la plupart des stages, suivant que l'entreprise se trouve en zone émettrice ou en zone d'accueil touristique;

- sous-représentation de certaines catégories d'entreprises inhérente à la saison estivale qui est celle des stages: secteur des loisirs urbains... et par définition ignorance totale de l'activité sports d'hiver!

- dans certains cas, indifférence réciproque des stagiaires et des entreprises, le stage étant vécu comme une obligation pour les uns, un service rendu à des établissements scolaires pour les autres.

Comment améliorer globalement l'exploitation pédagogique du stage au cours d'un cycle de formation? Plusieurs pistes peuvent être explorées, certaines le sont déjà:

- des stages assortis à un projet dans l'entreprise, que ce soit dans le cadre d'une réflexion globale sur la place d'un stagiaire dans l'entreprise ou d'un projet précis (enquête à mener ou autre...);

- un encadrement et un suivi du centre de formation pendant le stage, pouvant se traduire par des visites, lesquelles contribuent toujours à améliorer la communication entre formateurs et entreprises;

- des accords contractuels ne se limitant pas au statut du stagiaire mais s"étendant à son insertion, à une "charte de qualite" du stage;

- des stages non coupés du reste de la formation (stages en cours de cycle et non à la fin, stages éventuellement fragmentês, etc.);

- un véritable mémoire de stage qui pourrait être inséré dans une recherche individuelle du stagiaire, un projet précis et ceci quel que soit le niveau de formation, une telle démarche n'étant pas une exclusivité de l'université;
- des stages ne se limitant pas au territoire national mais s'étendant à l'Europe. Le problème de l'encadrement des stagiaires pourrait êttre réglé par des "échanges de suivi des stages " avec des établissements étrangers: la coopération en ce domaine pourrait être fructueuse.

Ces remarques vont dans le sens d'une plus grande importance accordée aux stages, laquelle va de pair avec une plus grande attention accordée à leur finalité et aux modalités de leur déroulement.

Une réflexion des formateurs sur les moyens disponibles aujourd hui ne doit pas dispenser d'une démarche similaire quant à la finalité des formations et à leurs résultats. Elle ne peut non plus êttre menée isolément, coupée des autres catégories d'intéressés: ainsi, il faudra bien progresser en direction d'une confiance mutuelle dans les fonctions des formateurs et milieux professionnels du tourisme et des loisirs. Chacun a besoin des autres et si les formations intégrées à l'entreprise restent l'apanage des grands groupes, même ceux-ci ont besoin de futurs collaborateurs bénéficiant d'une bonne formation initiale.

La formulation précise des constats aide a celle des propositions, c'est une évidence, mais une barrière sépare toujours ces deux niveaux: celle justement qui est constituée par les moyens disponibles. La faiblesse de ceux-ci rend a priori certaines propositions "irréalistes" et leur formulation devient inutile: pourquoi, alors, mener une réflexion?

Mais si les formateurs ne réflechissent pas individuellement et collectivement sur leur métier, les risques de déphasage sont grands d'autant plus que les evvolutions intervenant dans leurs activités proviennent quelquefois de l'extérieur et sont difficilement perceptibles, Ainsi, les "Dossiers de l'Éndiant" jouent un certain rôle dans le marché de la formation touristique en publiant chaque année les résultats d'établissements proposant des candidats aux examens débouchant sur des BTS du tertiaire, dont le BTS Tourisme. Cette transparence de l'information est forcément positive; avec quels résultats? Moralisation du marché? Développement d'une attitude de consommateurs de formation? En tout cas, le problème des grilles d'évaluation des formations est posé.

La coupure entre formation initiale et continue doit aussi faire l'objet d'une réflexion: l'obtention d'un diplôme de type " $b a c+2$ ", "bac +3 ", etc... ne peut pas constituer pour un individu l'investissement-formation définitif sur lequel il va asseoir sa carrière. Dans un secteur où la taille des entreprises est réduite et la mobilité des actifs est élevée. il faut aussi que la demande en formation continue vienne des intéressés, qu'ils gèrent leur carrière en intégrant la composante FORMATION, Comment les y aider? L'émiettement des offres de stages n'est pas forcément sécurisant pour cela. Pourquoi ne pourrait-on pas concevoir une formation continue composée d'unités capitalisables acquises

- dans des centres publics ou assimilés,

- dans des centres privés agréés avec des formations reconnues valables, et ceci sur l'ensemble du territoire national, puis européen?

Les moyens existent, leur seule mise en oeuvre cohérente est nécessaire.

Dernier thème de réflexion pour cet article: la nature des emplois créés par certains secteurs du tourisme et des loisirs. N'oublions pas que les terrains de golf génèrent de nombreux emplois de jardiniers et que les parcs de loisirs ont besoin d" "hommesorchestres" de l'animation. Le tourisme ne créant pas que des emplois "touristiques". il est indispensable d'identifier les emplois pour lesquels les formations touristiques peuvent réllement faire quelque chose.

\begin{abstract}
Références
I.N.S.E.E. Institut National de la Statistique et des Etudes Economiques]: dossier L'entrée des jeunes dans la vie active, Economie et Statistiques no 216 , decembre 1988, p. 23 aे 59.

LEHALLLE Dominique; European Institute of Tourism Management - La grande 6 cole, Echo Touristique no 40. 21 novembre 1988

ROUGE Marianne: Les L.E.A. jouent l'Europe, Le Monde, 2 février 1989

RUFFIN Françoise: La diversité des formations aux métiers du tourisme, collogue L'enjeu touristique, Ed. Economca, 1988, р. 259 a 266 .
\end{abstract}

\section{(Suite de la page 33 )}

les tarifs de 20 à $25 \%$ entre le Québec et lat France.

\section{Gouvernement muet}

Les autorités gouvernementales québécoises sont restées muettes dans ce dossier. Pourtant, le fait de limiter l'offre risque de faire monter les prix. Pour qui se préoccupe de réduire le déficit de la balance des échanges touristiques, une telle mesure est positive parce que de nature à dissuader les gens de se rendre en Europe. Tout aussi logiquement, ceci pourrait refroidir les ardeurs des Français dans leur désir de venir sillonner le Québec. Les années qui viennent seront donc décisives en ce qui concerne l'aboutissement de ce dossier. Les velléités protectionnistes de la France sont connues en matière de transport aérien, mềne si elles sont souvent contraires aux intérêts de son tourisme.

A l'inverse, la politique de déréglementation d'Ottawa - à l'intérieur d'abord puis répercutée dans le même esprit dans les relations bilatérales - semblait tout aussi évidente. Mais il va falloir attendre entre deux ans pour s'en convaincre que prennent fin les règlements actuels régissant les vols CanadaFrance. 